\title{
Papillary Tumor of the Temporal Bone
}

\author{
Bernhard Schick, M.D., 1 Hartmut Kronsbein, M.D.,2 Gabriele Kahle, M.D.,3 \\ Andreas Prescher, M.D.,4 and Wolfgang Draf, M.D., D.M.Sc., F.R.C.S.5
}

\begin{abstract}
Papillary tumors of the middle and inner ear have been interpreted histogenetically in many ways. In 1989 Heffner proposed the endolymphatic sac epithelium as a possible origin. These rare tumors are clinically aggressive and can cause extensive temporal bone destruction. Because of this behavior, endolymphatic sac tumors (ELST) were classified as low-grade adenocarcinomas, although metastasis has not yet been documented. Two papillary neoplasms of the temporal bone are presented, which we believe are examples of adenomatous tumors arising from the epithelium of the endolymphatic sac. One was associated with a pituitary adenoma. A third case of a papillary middle ear neoplasm is described that shows histologic features similar to the other two, but it was located in the tympanum and had no connection to the endolymphatic sac. This report focuses on clinical, radiologic, and histologic findings of papillary tumors of the temporal bone with additional emphasis on modern concepts of histogenesis and aspects of differential diagnosis.
\end{abstract}

KEYWORDS: Endolymphatic sac pathology, temporal bone tumor, Heffner tumor, papillary adenoma

\begin{abstract}
As in any other site, tumors of the temporal bone must be divided into local primaries, contiguous spread of adjacent neoplasms, and metastasis. Histomorphologically, papillary variants of renal
\end{abstract}

cell cancer, thyroid carcinoma, and choroid plexus tumors can be very similar to primary papillary tumors of the temporal bone (PTTB). Immunohistologically, these tumors share coexpression of $\mathrm{cy}-$

Skull Base, volume 11, number 1, 2001. Reprint requests: Bernhard Schick, M.D., ENT Department, University of Homburg, Kirrberger Str., D-66421 Homburg/Saarland, Germany. E-mail: hnobsch@med-rz.uni-saarland.de. ${ }^{1}$ ENT Department, University of Homburg, Homburg/Saarland, Germany; ${ }^{2}$ Institute of Pathology, ${ }^{3}$ Institute of Radiology, Hospital Fulda, Academic Teaching Hospital of the University of Marburg, Fulda, Germany; ${ }^{4}$ Institute of Anatomy and Embryology, RWTH Aachen, Germany; ${ }^{5}$ Department of Ear, Nose and Throat Diseases, Head, Neck, and Facial Plastic Surgery, Communication Disorders, Hospital Fulda, Academic Teaching Hospital of the University of Marburg, Fulda, Germany. Copyright (C) 2001 by Thieme Medical Publishers, Inc., 333 Seventh Avenue, New York, NY 10001, USA. Tel: +1(212) 584-4662. 1531-5010,p;2001,11,01,025,034,ftx,en; sbs00204x. 
tokeratins and vimentin and, with the exception of renal cell cancer, the expression of neuron-specific enolase (NSE).

Pure papillary adenomas of the temporal bone were reported to be more aggressive, causing bone destruction and intracranial extension, compared with mixed adenomas. ${ }^{1-3}$ The term "aggressive papillary middle ear tumor" was suggested, ${ }^{2}$ which avoided a definitive statement as to the dignity and the histogenesis of this neoplasm. A modern and largely accepted interpretation of PTTB was given by Heffner, who found evidence that these tumors arise from the epithelium of the endolymphatic sac when he studied 20 cases from the files of the Armed Forces Institute of Pathology. ${ }^{3}$

This article focuses on the rare PTTB (Heffner tumor) that arises from the epithelium of the endolymphatic sac. Three cases of papillary tumors of the middle and inner ear are presented.

\section{MATERIALS AND METHODS}

All three case histories are from the files of the Ear, Nose, and Throat Department at Fulda, Germany, between 1985 and 1997. Case 1 has been previously reported in part. ${ }^{4}$ Initial histologic examinations were done with hematoxylin and eosin (H\&E) staining. Additional immunostaining was performed with alkaline phosphatase-anti-alkaline phosphatase (APAAP) technique, using antibodies from DAKO (Denmark) against cytokeratin 7 (OV-TL 12/30), cytokeratin 8 (35ßH11), cytokeratin 10/13 (DE-K13), cytokeratin 18 (DC10), cytokeratin 19 (RCK108), cytokeratin 20 (KS20.8), epithelial membrane antigen (EMA, E29), vimentin (Vim3B4), S-100 (protein S-100 de vache, poly), neuron-specific enolase (NSE) (BBS/NC /VI-H14), synaptophysin (SY38), and chromogranin (DAK-A3). Pancytokeratin antibody KL-1 was purchased from Immunotech. Monoclonal S-100 antibodies (15E2E2) were obtained from BioGenex.

\section{CASE REPORTS}

\section{Case 1}

A 32-year-old man presented in 1995 with a history of progressive hearing loss on the right side and intermittent episodes of vertigo, which had begun 10 years earlier. Because of recurrent episodes of sudden deafness with different rates of recovery after symptomatic treatment, magnetic resonance imaging (MRI) was performed in 1990. The radiologic report described a small tumor between the internal auditory canal and the sigmoid sinus at the posterior surface of the temporal bone, which was interpreted by the radiologist as a small meningioma; however, this tumor was not considered responsible for the symptoms. In 1995, computed tomography (CT) identified a neoplasm $2 \mathrm{~cm}$ in diameter, which had led to temporal bone destruction (Fig. 1A), and magnetic resonance imaging (MRI) delineated the solid and cystic tumor portions (Fig. 1B,C). CT and MRI pinpointed the region of the endolymphatic sac as the central position of the tumor. Angiography demonstrated a vascularized tumor with blood supply arising from the ascending pharyngeal artery (Fig. 1D).

The tumor was resected using a retrolabyrinthine presigmoidal approach. Eight weeks after the operation, MRI showed no evidence of residual tumor. At 1-year follow-up, the patient had no complaint, but MRI indicated a tumor recurrence measuring $2.5 \times 1.5 \mathrm{~cm}$ (Fig. 1E). The mainly cystic and intradural neoplasm extended from the tentorium to the jugular foramen. This tumor recurrence was resected in our Neurosurgical Department (by Prof. Dr. Th. Wallenfang), using a suboccipital approach. During surgery, the caudal cranial nerves were found to have been displaced by the mainly cystic tumor. Solid tissue parts of the neoplasm were located at the surface of the petrous bone. Postoperative temporary weakness of the abducent nerve, with double vision, was noted. MRI performed 22 months after revision surgery indicated no tumor recurrence. 

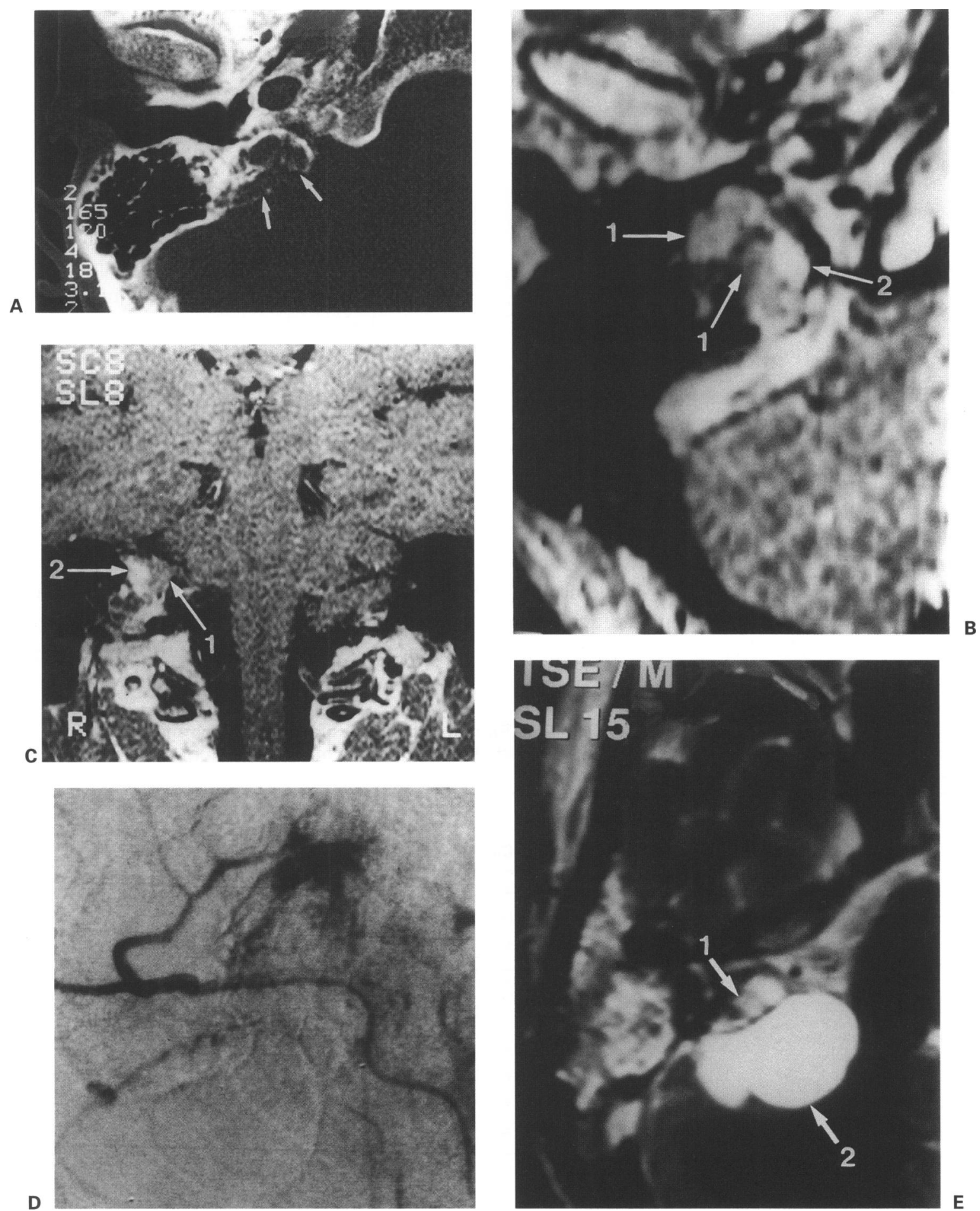

Figure 1. Axial CT scan (A) showing temporal bone destruction in a 32-year-old man. Solid (1) and cystic (2) tumor areas are evident in axial (B) and coronal (C) MRI. Angiography (D) shows tumor blood supply from the ascending pharyngeal artery. Axial MRI (E) 1 year after tumor resection indicates a tumor recurrence (1) with predominantly cystic portions (2). 


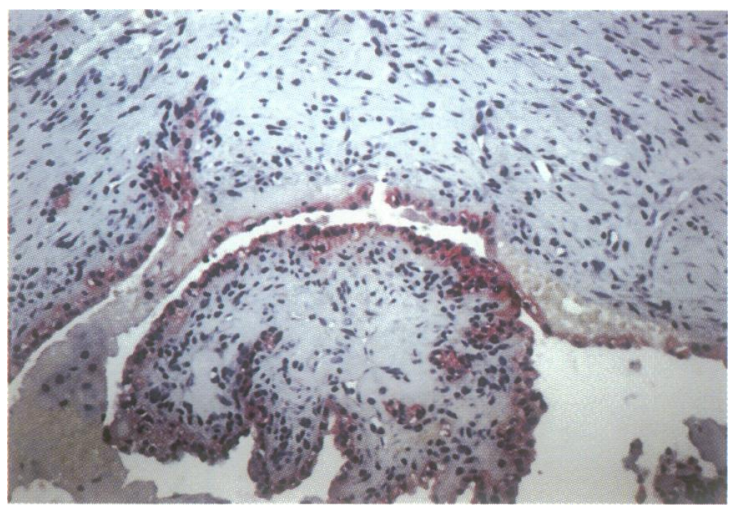

Figure 2. Papillary structure and fibrovascular stroma in the papillary-cystic temporal bone neoplasm of patient 1. The epithelium shows neuron-specific enolase immunoreactivity.

Histopathologic examination of a frozen section taken during the first surgery indicated a papillary tumor (Fig. 2). Microscopically, the tumor showed typical features of a papillary adenoma with cystic changes, as well as some bony infiltration. A single layer of cylindrical epithelial cells lined the mostly dense papillary stroma. There was no difference in primary and recurrent tumor. The immunohistologic results are presented in Table 1.

\section{Case 2}

A 66-year-old man was suffering from chronic otitis media after exposure to a bomb explosion during World War II. Mastoidectomy was performed during the 1940s, and in 1973, a cholesteatoma was surgically treated by revision tympanoplasty type III. Recurrence of a cholesteatoma was again treated surgically in 1982 . During surgery, a fistula in the lateral semicircular canal was noted. It was also observed that the dura of the middle skull base was not totally covered by bone. In 1989, the patient developed a peripheral paralysis of the right facial nerve. CT showed a tumor of the pituitary gland and a second one destroying the entire temporal bone with extension into the posterior and middle cranial fossae (Fig. 3).

Table 1 Immunohistologic Results of Three Papillary Tumors of the Temporal Bone in Comparison with Immunohistologic Findings in Endolymphatic Sac Epithelium and Endolymphatic Sac Tumors Reported in the Literature

\begin{tabular}{|c|c|c|c|c|c|}
\hline & Case 1 & Case 2 & Case 3 & $\begin{array}{l}\text { Findings in } 64 \\
\text { Endolymphatic } \\
\text { Sac Specimens } 25\end{array}$ & $\begin{array}{l}\text { Experiences in } \\
23 \text { Endolymphatic } \\
\text { Sac Tumors? }\end{array}$ \\
\hline CK KL-1 & + & ++ & + & ND & $23 / 23(100 \%)$ \\
\hline CK 7 & + & ++ & $(+)$ & $++^{a}$ & ND \\
\hline CK 8 & $(+)$ & ++ & + & $++^{a}$ & ND \\
\hline CK $10 / 12$ & - & - & - & $++^{b}$ & ND \\
\hline CK 18 & - & - & $(+)$ & +++ & ND \\
\hline CK 19 & + & ++ & - & +++ & ND \\
\hline CK 20 & - & - & - & ND & ND \\
\hline Vimentin & + & + & + & +++ & $9 / 9(100 \%)$ \\
\hline NSE & + & + & + & + & $6 / 7(86 \%)$ \\
\hline Synaptophysin & - & - & - & ND & ND \\
\hline Chromogranin & - & - & - & + & ND \\
\hline S-100 poly & + & + & + & +++ & $14 / 23(61 \%)$ \\
\hline S-100 mono & - & - & - & ND & ND \\
\hline EMA & + & + & + & + & $4 / 4(100 \%)$ \\
\hline
\end{tabular}

CK, cytokeratin; NSE, neuron-specific enolase; EMA, epithelial membrane antigen; poly, polyclonal; mono, monoclonal; ND, no data. aAnti-cytokeratin 1-8 used.

${ }^{b}$ Anti-cytokeratin 13 used. 


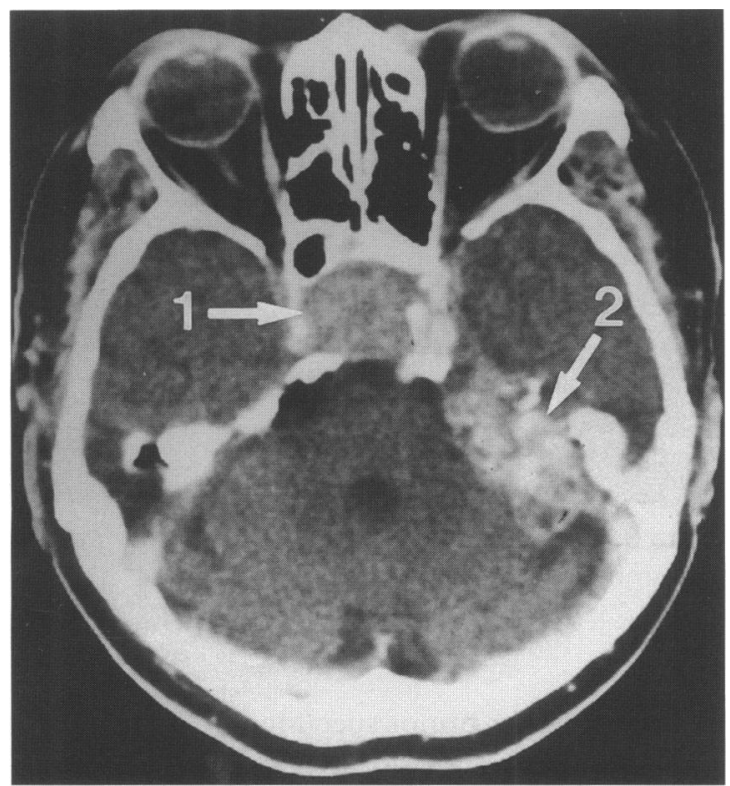

Figure 3. Axial CT scan showing a pituitary gland tumor (1) and a second large neoplasm (2) with nearly complete temporal bone destruction in a 66-year-old man.

Angiography showed a hypervascular neoplasm with blood supply arising from the ascending pharyngeal, greater auricular, superficial temporal, and occipital arteries. The radiologic findings were believed to indicate a paraganglioma in the region of the temporal bone.
The temporal bone neoplasm was resected by a complete petrosectomy. The vertical and horizontal parts of the internal carotid artery were embedded in the tumor, but they could be clearly dissected from it. Postoperatively, the patient suffered a cerebral infarction and unfortunately died 4 weeks after the operation.

Intraoperative frozen-section examination showed a papillary adenomatous tumor, very similar to a well-differentiated papillary thyroid cancer, with cystic portions and areas of haemorrhage and sclerosis. The macroscopic appearance of the resected specimen $(4.5 \times 3 \times 2 \mathrm{~cm})$ of the petrous bone showed predominantly inhomogeneous yellowbrown tissue. Histologic examination (Fig. 4A,B) showed findings similar to those of the frozen section biopsy. The arborized fibrovascular stroma was lined with tall columnar cells, which appeared to push the borders, rather than invade the tissue. The immunohistologic results are enumerated in Table 1. Postmortem examination revealed a subtotal infarction of the right cerebral hemisphere and myocardial infarction of the ventricular septum. Minor remnants of the papillary tumor were seen only in the vicinity of the carotid canal.

The pituitary gland tumor $(3 \mathrm{~cm}$ in diameter) consisted mainly of rather clear cells with a
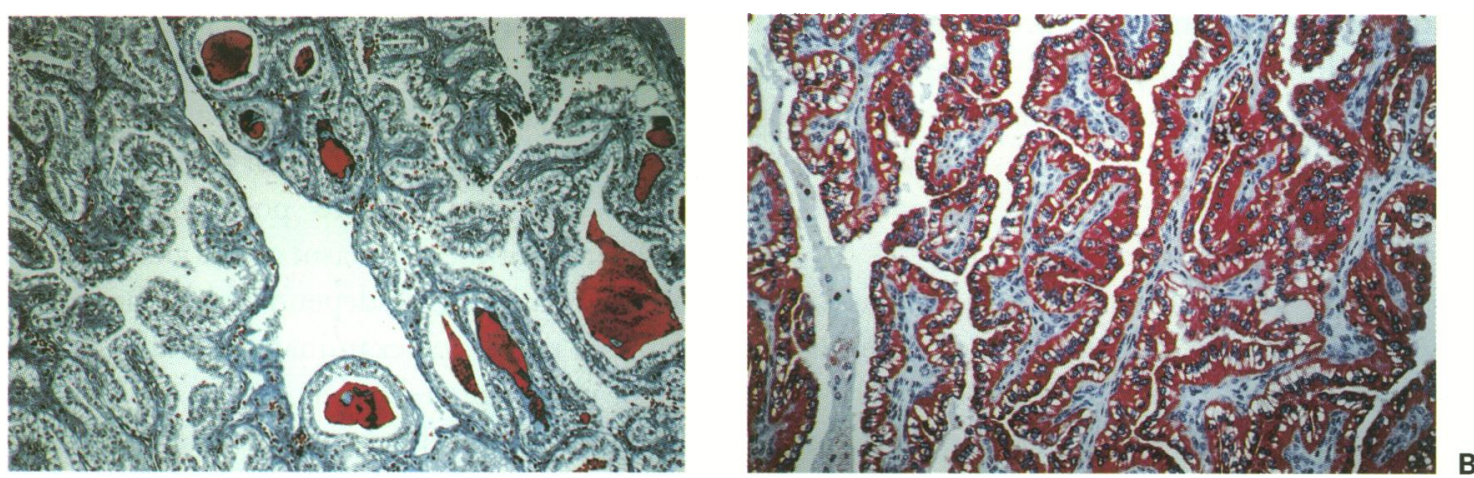

Figure 4. Papillary temporal bone tumor probably originating from the epithelium of the endolymphatic sac in patient 2. This case has an aspect of colloid-filled cavities (A), which can lead to misdiagnosis of metastatic thyroid carcinoma (Masson trichrome stain). Strong immunoreactivity of this endolymphatic sac tumor with anticytokeratin 7 antibody (B). 
solid growth pattern. Immunohistopathologically, the tumor was characterized as a large-cell chromophobic pituitary adenoma with positive reaction to cytokeratin, NSE, chromogranin A, and synaptophysin. To summarize, the skull base of this patient had been invaded by two different tumors that met in the region of the carotid canal, a rare papillary adenoma of the temporal bone and a typical pituitary adenoma.

\section{Case 3}

A 29-year-old woman complained in 1985 of a slight hearing loss of the right ear. Otoscopic examination showed a reddish tumor shimmering through an intact eardrum in the upper quadrant, and audiometry proved a slight conductive hearing loss. CT exhibited soft tissue around the incus. The mastoid space was aerated, and no opacification was observed. Because of the clinical and radiologic findings, the operation was performed in a tympanoplastic-like manner. A low vascularized tumor encapsulating the incus and extending into the sinus tympani and the hypotympanum was removed. Bone destruction was not observed during surgery. Intraoperative frozen-section analysis indicated a glandular neoplasm of uncertain dignity. Histopathologic examination with expert consultation led in 1985 to the diagnosis of a low-grade adenocarcinoma of the middle ear. Therefore, resection of the petrous bone preserving the facial nerve and inner ear function, a suprahyoidal neck dissection, and a total parotidectomy were performed in a second operation. Postoperative recovery was uneventful. No recurrence was detected at a 12-year follow-up examination.

Histologically, the tumor appeared as a papillary adenoma (Fig. 5) with features similar to those seen in cases 1 and 2 . In contrast to the other two cases, no cystic changes were seen. The results of the recently conducted immunohistologic investigations are summarized in Table 1 . Tissue removed during the second surgical treatment re-

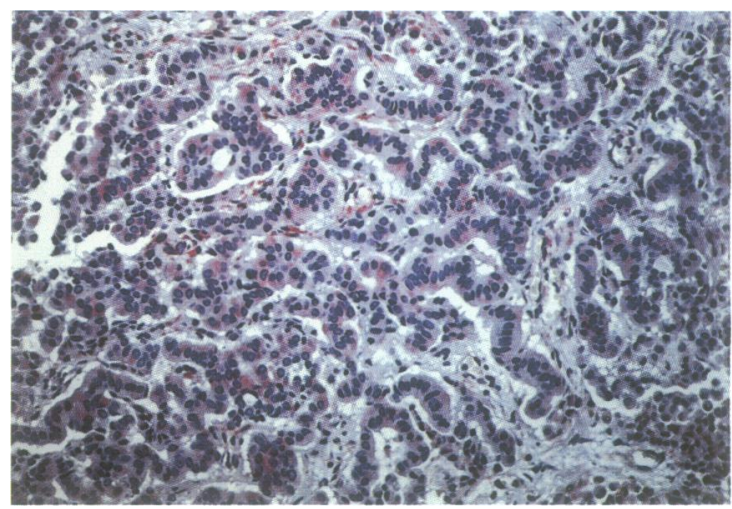

Figure 5. Papillary tumor of the middle ear in patient 3 with vimentin immunoreactivity.

vealed no further tumor specimen on histologic examination. Based on the finding of tumor extension limited to the middle ear space without signs of bone destruction and the actual histologic reexamination, the tumor is reclassified as a papillary middle ear adenoma.

\section{DIsCussion}

In 1989, Heffner described a papillary neoplasm, probably originating from the epithelium of the endolymphatic sac as an independent entity based on clinical, radiologic, immunohistologic, and electron microscopic findings. ${ }^{3}$ Clinically and radiologically, the papillary tumor is characterized by (1) locally aggressive growth with temporal bone destruction and, in most cases, a long clinical prodrome; (2) late recognition of the tumor frequently with extension to the posterior cranial fossa; (3) center of the neoplasm at the vicinity of the endolymphatic sac, independent of the size at the time of radiologic examination; and (4) association with von Hippel-Lindau disease in 15\% of cases, with possible manifestation of bilateral tumor. 3,5

The theory that PTTB originate from the epithelium of the endolymphatic sac was supported by different investigators based on the clini- 
cal and radiologic presentation of these tumors and by histopathologic and immunohistochemical similarities between the neoplasm and normal epithelium of the endolymphatic sac.5,6 The identification of a de novo papillary epithelial lesion arising within the confines of the endolymphatic sac and endolymphatic duct was reported by Megerian et al. ${ }^{7}$ in an autopsy of a patient with von HippelLindau disease. This patient had a large, destructive papillary neoplasm in the opposite temporal bone. Immunohistochemical analysis in tissue culture from a PTTB provided further evidence that epithelium from the endolymphatic sac is the site of origin for this papillary neoplasm.6,8 Since 1989, classification of PTTB according to Heffner's concept was suggested in further observations of this rare tumor. ${ }^{9-13}$ Roche et al. ${ }^{14}$ found a total of 56 PTTB in the literature that were compatible with a papillary neoplasm arising from endolymphatic sac epithelium.

The clinical manifestations are hearing loss, vertigo, cerebellar disorders, and facial nerve paralysis. Almost all patients suffered from a loss of hearing, while facial nerve paralysis and cerebellar disorders were observed in $44 \%$ of patients. ${ }^{5}$ In the past, papillary neoplasms arising from endolymphatic sac epithelium often were not recognized until they were in advanced stage. The radiologic examination showed large tumors $(>4 \mathrm{~cm}$ in diameter) that extended into the posterior cranial fossa and caused temporal bone destruction., ${ }^{3,5}$ The blood supply in HEFFNER-tumors appears to come predominantly from the external carotid artery, ${ }^{15}$ as seen in cases 1 and 2.

Histologically, the endolymphatic sac tumor (ELST) appears as a papillary cystic neoplasm often resembling thyroid cancer. The papillary portion of the tumor may be small, but it is especially important for distinguishing from middle ear adenomas, which usually do not present with papillary architecture. In contrast to paragangliomas, the PTTB are unquestionably of epithelial origin. Microscopic similarities between papillary neoplasms arising from the endolymphatic sac and the choroid plexus can further complicate diagnosis. The endolymphatic sac and the choroid plexus have the same neuroectodermal origin. Both present as well as mesothelium and thyroid epithelium endoepithelial tissue, which line inner cavities, fulfilling secretory and resorptive functions. ${ }^{16}$ Tumors arising from these endoepithelial tissues can show similar histologic appearance resembling papillary architecture. Immunohistochemically, these tumors express cytokeratins, vimentin, and neuroendocrine markers. Transthyretin staining was recommended to distinguish between tumors originating from the endolymphatic sac and the choroid plexus, as this marker is more likely to be found in choroid plexus epithelium and should be absent in endolymphatic sac tumors. ${ }^{17}$

On the basis of local aggressive growth with bone destruction, Heffner ${ }^{3}$ classified the tumor in 1989 as a low-grade adenocarcinoma, although metastasis has not yet been reported. More recently, Heffner preferred the term "invasive papillary cystadenoma of endolymphatic sac origin" for these lesions. ${ }^{18}$ The World Health Organization $(\mathrm{WHO})^{19}$ classifies the tumor as both adenoma and adenocarcinoma of the middle and inner ear. Classifying PTTB as low-grade adenocarcinoma is questionable because most tumors that develop in or near the temporal bone will lead to bone destruction, regardless of their nature. Paragangliomas or pituitary gland tumors would not be classified as malignant simply on the basis of the observation of erosion, which is clearly demonstrated by the second case.

Regardless of the biologic nature of the tumor, one should keep in mind the locally destructive behavior of ELST. The tumor recurrence in case 1 and the extensive temporal bone destruction in case 2 emphasize the aggressive local tumor growth, which confronts the surgeon with the difficulties of complete tumor resection. Total tumor removal is important, but it may be impossible in advanced stages. ${ }^{14}$ Therefore, appropriate treatment consists of detection of PTTB in its early stages, preoperative diagnosis of ELST on the basis 
of radiologic findings, and selection of a surgical approach that will allow removal of the tumor. PTTB grow slowly, so careful follow-up is important to detect tumor recurrence. Few experiences with radiotherapy are available, ${ }^{14}$ but this is not believed to be an effective treatment option.

Case 1 has all the aspects of a typical ELST. The origin of the lesion in the area of the endolymphatic sac was documented radiologically 5 years before diagnosis. Although it is difficult to pinpoint the origin of a late-stage tumor retrospectively, the clinical, radiologic, and histologic findings indicate that the temporal bone tumor in the second case can also be classified as a papillary neoplasm originating from the endolymphatic sac epithelium. This would be the first report of a ELST combined with a pituitary adenoma. The third case is described here to demonstrate that papillary differentiation can be observed in rare cases of middle ear tumors. Papillary tumors of the middle ear probably arise from neural crest cells in the middle ear. ${ }^{20}$ From a clinical point of view (conductive hearing loss, tympanal location, no bone destruction) the papillary neoplasm in the third case showed no similarities to a ELST. In addition, on the immunohistologic level a different pattern of cytokeratin expression was noted.

The supposition that all papillary tumors of the middle and inner ear originate from the endolymphatic sac epithelium was questioned by Pollak et al., ${ }^{21}$ who described a papillary adenoma originating from the mucosa of the pneumatic spaces surrounding the jugular bulb. This report was criticized, ${ }^{22,23}$ but it raises the issue of correct histogenetic interpretation of PTTB, as well as different theories of origin from adenomatous temporal bone tumors. ${ }^{24}$

Recent publications support the origin of papillary lesions of the temporal bone from endolymphatic sac epithelium. 3,5-7,9,10,12-14,17,20,22,23 Our experience demonstrates the importance of using clinical, radiologic, and histologic findings in diagnosing a papillary neoplasm as arising from the endolymphatic sac epithelium. Papillary growth pattern alone does not justify classification of an adenomatous lesion of the temporal bone as a ELST, particularly because tumors arising from the endoepithelium as endolymphatic sac, choroid plexus, mesothelium, and thyroid epithelium can share immunohistochemical similarities besides papillary architecture.

\section{REFERENCES}

1. Benecke JE, Noel FL, et al. Adenomatous tumors of the middle ear and mastoid. Am J Otol 1990;11:20-26

2. Gaffey MJ, Mills SE, Fechner RE, et al. Aggressive papillary middle-ear tumor: a clinicopathologic entity distinct from middle-ear adenoma. Am J Surg Pathol 1988;12: 790-797

3. Heffner D. Low-grade adenocarcinoma of probable endolymphatic sac origin: a clinico-pathologic study of 20 cases. Cancer 1989;64:2292-2302

4. Schick B, Kahle G, Kronsbein H, Draf W. Papillärer Tumor des Saccus endolymphaticus. Head Neck Otolaryngol 1996;44:329-332

5. Lavoie M, Morency RM. Low-grade papillary adenomatous tumors of the temporal bone: report of two cases and review of the literature. Mod Pathol 1995;8:604-608

6. Feghali JG, Levin RJ, Llena J, et al. Aggressive papillary tumors of the endolymphatic sac: clinical and tissue culture characteristics. Am J Otol 1995;16:778-782

7. Megerian CA, McKenna MJ, Nuss RC, et al. Endolymphatic sac tumors: histopathologic confirmation, clinical characterization, and implication in von Hippel-Lindau disease. Laryngoscope 1995;105:801-808

8. Levin RJ, Feghali JG, Morganstern N, et al. Aggressive papillary tumors of the temporal bone: an immunohistochemical analysis in tissue culture. Laryngoscope 1996; 106:144-147

9. Batsakis JG, El-Naggar AK. Papillary neoplasms (Heffner's tumors) of the endolymphatic sac. Ann Otol Rhinol Laryngol 1993;102:648-651

10. Delisle MB, Uro E, Roequette I, et al. Papillary neoplasm of the endolymphatic sac in a patient with von HippelLindau disease. J Clin Pathol 1994;47:959-961

11. Li JC, Brackmann DE, Lo WM, et al. Reclassification of aggressive adenomatous mastoid neoplasms as endolymphatic sac tumors. Laryngoscope 1993;103:1342-1348

12. Meyer JR, Gebarski SS, Blaivas M. Cerebellopontine angle invasive papillary cystadenolymphoma of endolymphatic sac origin with temporal bone involvement. AJNR Am J Neuroradiol 1993;14:1319-1323

13. Poe DD, Tarlov EC, Thomas CB, Kveton JF. Aggressive 
papillary tumors of the temporal bone. Otolaryngol Head Neck Surg 1993;108: 80-86

14. Roche PH, Dufor H, Figarella-Branger D, Pellet W. Endolymphatic sac tumors: report of three cases. Neurosurgery $1998 ; 42: 927-932$

15. Mukherji SK, Albernaz VS, Lo WW, et al. Papillary endolymphatic sac tumors: CT, MR imaging, and angiographic findings in 20 patients. Radiology 1997;202: 801-808

16. Benninghoff A. Anatomie: makroskopische Anatomie, Embryologie und Histologie des Menschen. In: Drenckhahn D, Zenker W, eds. Vol 1. Munich: Urban \& Schwarzenberg; 1994:114

17. Megerian CA, Pilch BZ, Bhan AK, McKenna MJ. Differential expression of transthyretin in papillary tumors of the endolymphatic sac and choroid plexus. Laryngoscope 1997;107:216-221

18. Wenig BM. Schneiderian-type mucosal papillomas of the middle ear and mastoid. Ann Otol Rhinol Laryngol 1996;105:226-233

19. Shanmugaratnam K, Sobin LH. Histological typing of tu- mours of the upper respiratory tract and ear. Berlin: World Health Organization/Springer; 1991:26

20. Kempermann G, Neumann HPH, Volk B. Endolymphatic sac tumours. Histopathology 1998;33:2-10

21. Pollak A, Böhmer A, Spycher M, Fisch U. Are papillary adenomas endolymphatic sac tumors? Ann Otol Rhinol Laryngol 1995;104:613-619

22. Heffner D. Are papillary adenomas endolymphatic sac tumors [letter]? Ann Otol Rhinol Laryngol 1996;105: 251-252

23. Megerian CA. Are papillary adenomas endolymphatic sac tumors [letter]? Ann Otol Rhinol Laryngol 1996;105: 83-84

24. Bold EL, Wanamaker JR, Hughes GB, et al. Adenomatous lesions of the temporal bone. Immunohistochemical analysis and theories of histogenesis. Am J Otol 1995;16: 146-152

25. Altermatt HJ, Gebbers JO, Müller C, Laissue J. Immunohistochemical characterization of the human endolymphatic sac and its associated cell populations. Acta Otolaryngol 1992;112:299-305 